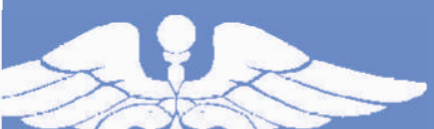

ISSN: 2782-7550 (Print) ISSN: 2782-7542 (Online)
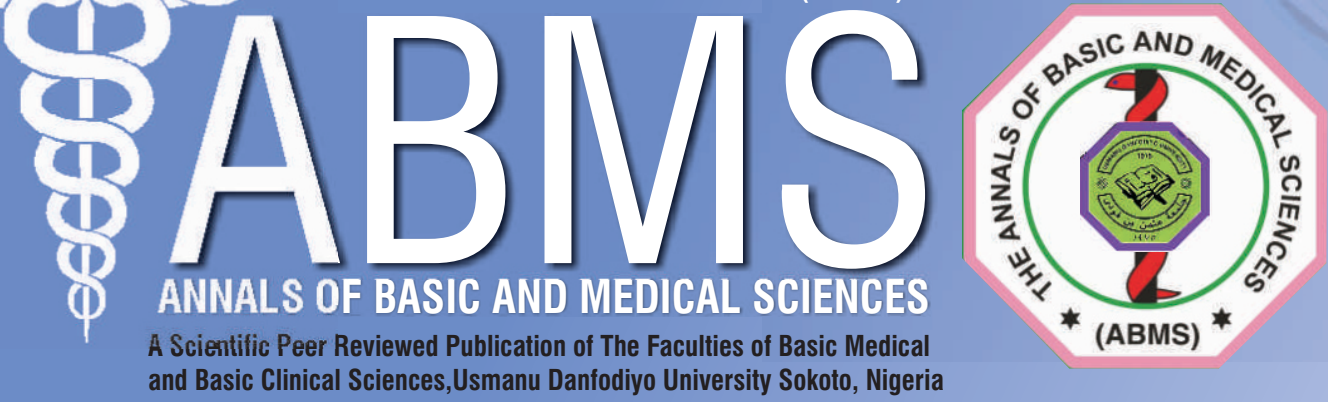

and Basic Clinical Sciences,Usmanu Danfodiyo University Sokoto, Nigeria
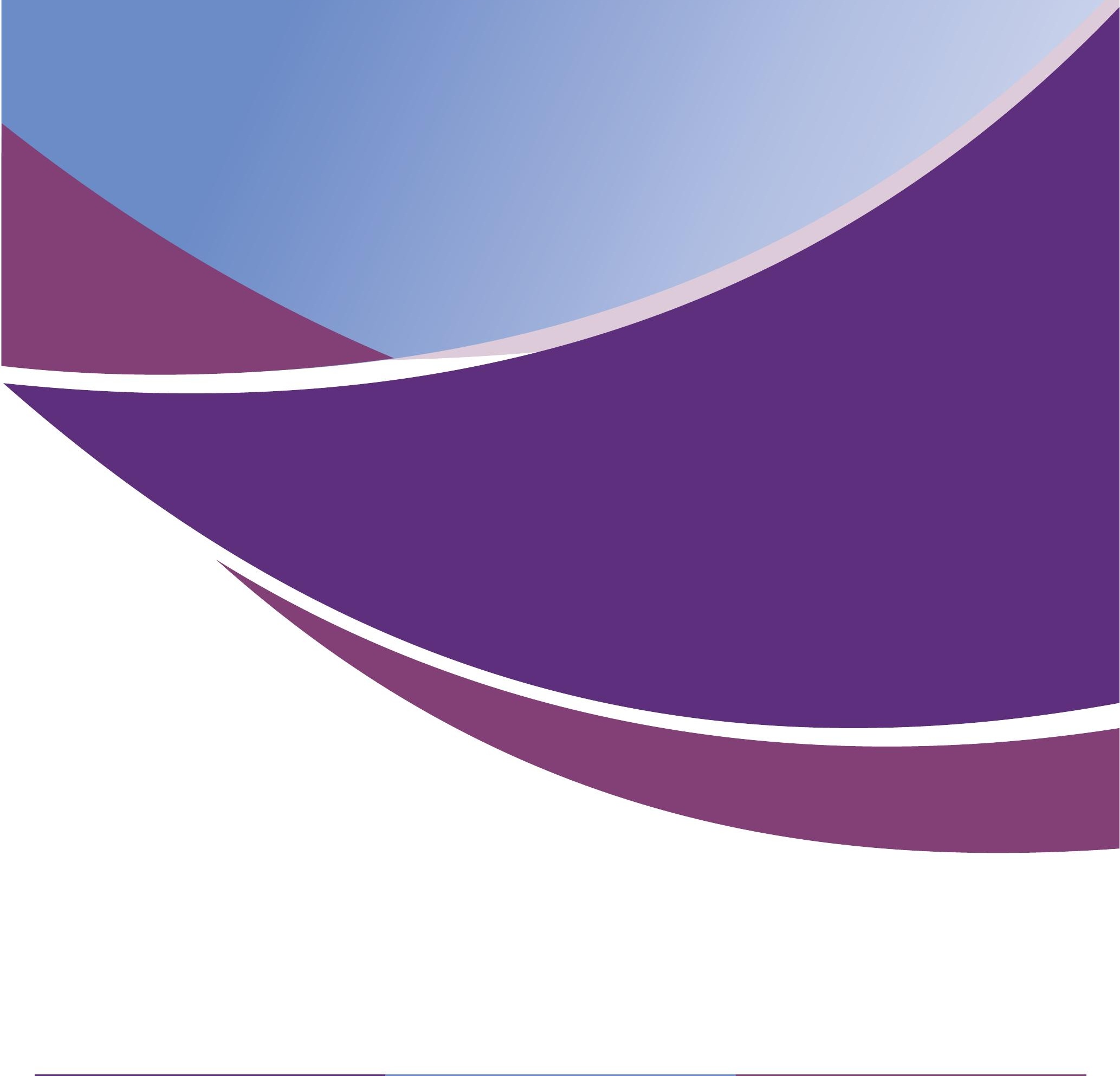

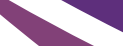
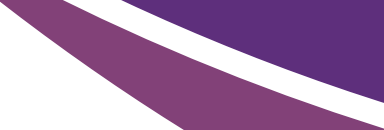


\title{
Phenotypic Detection of Methicillin and Inducible Clindamycin Resistant Staphylococcus Aureus: An Effort to Combat Antimicrobial Resistance and Improve Patient Safety in Gombe, Nigeria
}

\author{
Mohammed M Manga', Gloria O Michael ${ }^{2}$, Aishatu A Julde², Gidado Muhammad², \\ Umar M Hassan', Mohammed Ibrahim', Mohammed A Wulgo ${ }^{3}$, Elon W Isaac ${ }^{4}$
}

'Department of Medical Microbiology and Immunology, Gombe State University/Federal Teaching Hospital, Gombe, Nigeria

${ }^{2}$ Department of Medical Microbiology and Immunology, Federal Teaching Hospital, Gombe, Nigeria

${ }^{3}$ Department of Clinical Pharmacology and Therapeutics, Gombe State University, Gombe, Nigeria ${ }^{4}$ Department of Paediatrics, Gombe State University/Federal Teaching Hospital, Gombe

\begin{abstract}
:
Introduction: Antimicrobial resistance (AMR) is a major threat to patient safety. Methicillin and inducible clindamycin resistant Staphylococcus aureus are important multidrug resistant organisms (MDROs). Timely reporting of MDROs is necessary for rational antibiotic prescription and in combating AMR. We present the prevalence and distribution of Methicillin and inducible clindamycin resistant (iCR) isolates of Staphylococcus aureus from Gombe Nigeria.

Materials and Methods: This descriptive cross-sectional study included 260 isolates of Staphylococcus aureus from clinical specimens in Federal Teaching Hospital Gombe (FTHG). Isolates identification was done using conventional biochemical methods. Methicillin resistance was detected by cefoxitin disc diffusion method while iCR isolates by erythromycin and clindamycin disc approximation test (D-test). Data analysis was done using SPSS version 23.0.
\end{abstract}

Results: Methicillin resistant Staphylococcus aureus (MRSA) was detected in 178 (68.5\%) isolates while 214 (82.3\%) were iCR (D-test positive). Among the MRSA, $87.1 \%$ were also iCR while $72.4 \%$ of the iCR isolates were MRSA. There was significant association between MRSA and iCR $(p=0.03)$, MRSA and clindamycin resistance $(p=0.05)$ and MRSA and erythromycin resistance $(p<0.01)$.

Conclusion: Prevalence of MDR Staphylococcus aureus is high in Gombe Nigeria. Antimicrobial stewardship programme (ASP) and good Infection Prevention and Control (IPC) are necessary in combating AMR and improving patient safety.

Keywords: Methicillin Resistant Staphylococcus aureus (MRSA), Inducible Clindamycin Resistant Staphylococcus aureus (iCRSA), Antimicrobial Resistance (AMR), Patient Safety, Nigeria

Corresponding author:

Mohammed M Manga

Department of Medical Microbiology and Immunology,

Gombe State University and Federal Teaching Hospital Gombe, Nigeria Email: drmmanga@gmail.com Phone number: +2348036781726
Introduction

The emergence of antimicrobial resistance (AMR) posed one of the greatest challenges to patient safety and modern medicine (1). Mortalities due to multidrug resistant pathogens have continued all over the globe with worsening indices in developing countries where regulations guiding the use and sale of antimicrobials are suboptimal (2). The economic impact of AMR has continued to exert negative effects on both countries and families due to increased cost of care (2). Staphylococcus aureus has remained one of the most common and clinically relevant bacterial pathogens responsible for both local and systemic infections in both community and healthcare settings (3). There is a growing prevalence of multidrug resistant Staphylococcus aureus such as iCR and MRSA across the globe leading to poor patient outcome with more difficult and expensive treatment options (3). Multidrug resistant (MDR) Staphylococcus aureus has been ranked by the World Health Organisation (WHO) as one of the high priority pathogens that urgently require newer drugs for treatment (4). The presence of MRSA and co-resistance with iCR pathogens is associated with worsening of clinical presentation and increased frequency of treatment failure (5).

Diagnostic stewardship relies on good relationship between clinical laboratories and other clinicians/clinics for accurate diagnosis that will guide in providing better patient care for the best outcome and patient safety (6). This has become more critical in infectious diseases in view of the growing global threat of AMR which remains a major challenge to IPC and patient safety. (6) Phenotypic detection of both MRSA and inducible clindamycin resistance could be easily and realistically carried in most medical microbiology laboratories in low resource setting like Nigeria at a minimal or no extra cost $(7,8)$. Most of the studies from Nigeria and other developing countries utilized phenotypic detection methods in identifying the MDR pathogens $(5,9-11)$. 
Although there were few reports on MDR Staphylococcus aureus from North Eastern Nigeria, virtually all the studies were carried about a decade ago and only on MRSA $(12,13)$. Many other studies some of which also reported findings on inducible clindamycin resistance were from other parts of Nigeria (11). This study reported the prevalence and relationship of MRSA and inducible clindamycin resistance among clinical isolates of Staphylococcus aureus in Gombe Nigeria.

\section{Methods}

A descriptive cross-sectional study was conducted using a total of 260 non-repeat isolates of Staphylococcus aureus from clinical specimens (urine, swabs, blood, aspirates and seminal fluids) received in the department of medical microbiology and immunology, FTHG between May and October 2020. Identification of Staphylococcus aureus was done manually using biochemical methods (Gram staining, catalase and coagulase tests) and methicillin resistance was detected by cefoxitin disc diffusion method and inducible clindamycin resistance by erythromycin and clindamycin disc approximation test (D-test). Isolates with cefoxitin zone of inhibition $\geq 22 \mathrm{~mm}$ was regarded as methicillin susceptible while those with $\leq 21 \mathrm{~mm}$ was interpreted as methicillin resistant (14). Inducible clindamycin resistant phenotypes were identified based on resistance to erythromycin and having a clindamycin zone $\geq 21 \mathrm{~mm}$ with a D-shaped zone (14). Testing and interpretations were all based on Clinical and Laboratory Standards Institute (CLSI) performance standards for antimicrobial susceptibility testing (14). Staphylococcus aureus strains ATCC 25923 (MSSA), ATCC 43300 (MRSA) and ATCC 25923 (D-test positive) were used for quality control based on CLSI guidelines (14).

Ethical clearance for the study was obtained from the research and ethics committee (REC) of FTHG.

Data analysis was done using statistical package for social sciences (SPSS) version 23.0 software. Chisquare test was used in analyzing categorical variables $(P<0.05$ was considered statistically significant).

\section{Results}

Of the 260 isolates, majority were from females (59.2\%) and more than two third (79.2\%) came from out-patients. More than half of the isolates were recovered from patients between the ages of 21 and 40 years (figure 1). Distribution of different resistant isolates based on type of specimen, gender of patients and origin of the isolates (in-patients and out-patients) were as highlighted in table 1 , figure 2 and figure 3 . Prevalence of MRSA was $68.5 \%$ while $82.3 \%$ were iCR. Among the MRSA, $87.1 \%$ were also iCR while $72.4 \%$ of iCR isolates were MRSA. Individual resistance to erythromycin and clindamycin was found among $86.5 \%$ and $85.8 \%$ of the isolates respectively. As shown in table 2 , there was significant association between MRSA and iCR isolates $(p=0.003)$, MRSA and individual clindamycin resistance $(p=0.005)$ and MRSA and individual erythromycin resistance $(p<0.001)$. There was no significant association between MRSA and origin of isolates (in-patients and out-patients) and gender of patients.

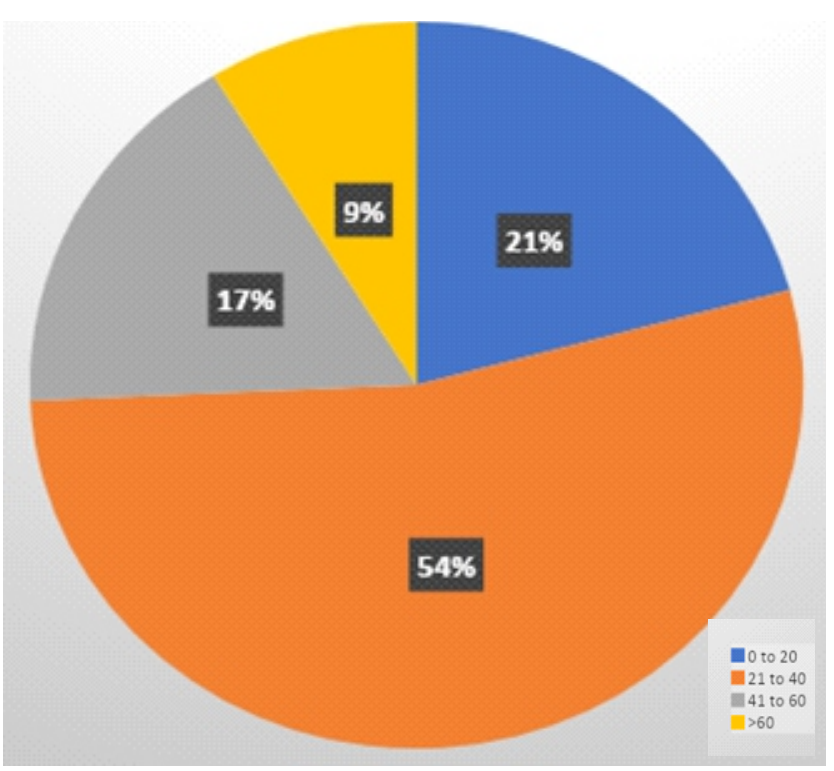

Figure 1: Age distribution of patients from whom the isolates were recovered.

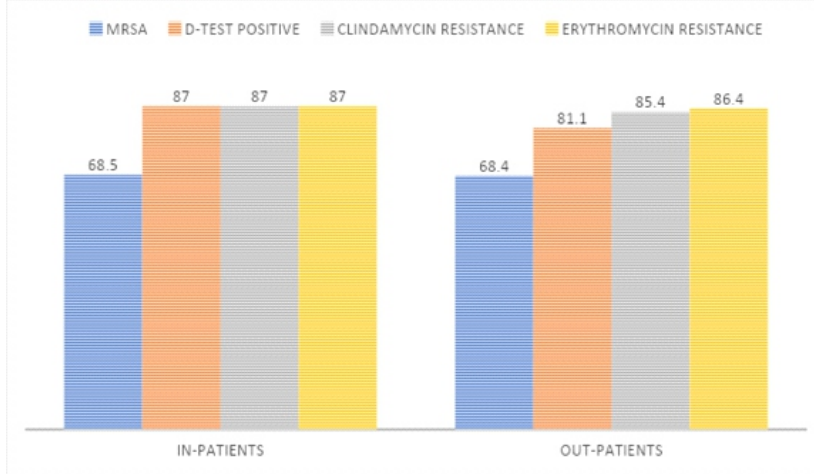

Figure 2: Pattern of resistance among isolates from inpatients and out-patients.

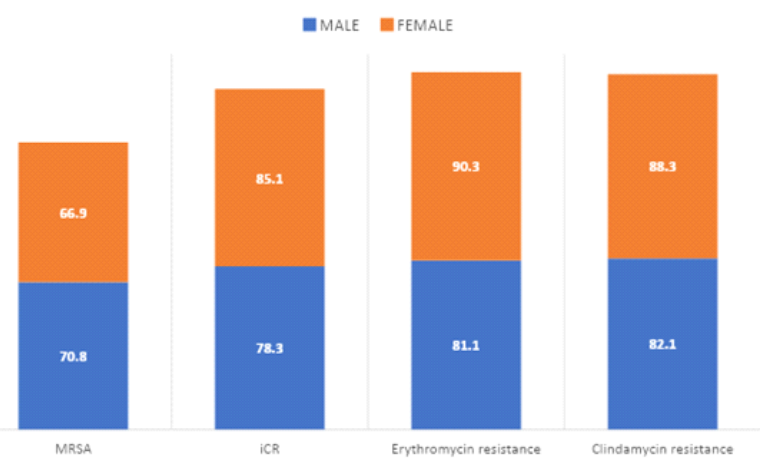

Figure 3: Pattern of resistance among isolates from males and females. 
Table 1: Distribution of different resistant isolates based on type of specimen

\begin{tabular}{llllll}
\hline Type of specimen & $\begin{array}{l}\text { Number of } \\
\text { isolates }(\%)\end{array}$ & MRSA & iCR & $\begin{array}{l}\text { Erythromycin Clindamycin } \\
\text { resistance } \\
\text { resistance }\end{array}$ \\
\hline Urine & $157(60.4)$ & $108(68.8)$ & $134(85.4)$ & $140(89.2)$ & $139(88.5)$ \\
Swabs & $47(18.1)$ & $30(63.8)$ & $34(72.3)$ & $35(74.4)$ & $37(78.7)$ \\
Seminal fluid & $22(8.5)$ & $17(73.3)$ & $20(90.9)$ & $22(100)$ & $20(90.9)$ \\
A spirates & $21(8.0)$ & $12(57.1)$ & $15(71.4)$ & $16(76.1)$ & $16(76.1)$ \\
Blood & $13(5.0)$ & $11(84.6)$ & $11(84.6)$ & $12(92.3)$ & $11(84.6)$ \\
\hline Total & & & & & $\mathbf{2 2 3 ( 8 5 . 8 )}$ \\
\hline
\end{tabular}

Table 2: Association between MRSA, other resistant patterns (iCR, erythromycin resistance clindamycin resistance), of patients and origin of isolates (in-patients and out-patients)

\begin{tabular}{llllll}
\hline & MRSA $(\mathrm{N} ; \%)$ & $\begin{array}{l}\text { MSSA }(\mathrm{N} ; \\
\%)\end{array}$ & Total $(\mathrm{N} ; \%)$ & $X$ & $P$ value \\
\hline iCR (D-test positive) & $155(72.4)$ & $59(27.6)$ & $214(100)$ & 8.822 & 0.003 \\
No iCR (D-test negative) & $23(50.0)$ & $23(50.0)$ & $46(100)$ & & \\
Total & $178(68.5)$ & $82(31.5)$ & $260(100)$ & & \\
Erythromycin resistant & $165(73.3)$ & $60(26.7)$ & $225(100)$ & 18.373 & $<0.001$ \\
Erythromycin sensitive & $13(37.1)$ & $22(62.9)$ & $35(100)$ & & \\
Total & $178(68.5)$ & $82(31.5)$ & $260(100)$ & & \\
$\quad$ Clindamycin resistant & $160(71.7)$ & $63(28.3)$ & $223(100)$ & 7.843 & 0.005 \\
Clindamycin sensitive & $18(48.6)$ & $19(51.4)$ & $37(100)$ & & \\
Total & $178(68.5)$ & $82(31.5)$ & $260(100)$ & & \\
Gender & & & & & \\
Males & $75(70.8)$ & $31(29.2)$ & $106(100)$ & 0.436 & 0.509 \\
Females & $103(57.9)$ & $51(33.1)$ & $154(100)$ & & \\
Total & $178(68.5)$ & $82(31.5)$ & $260(100)$ & & \\
Source of isolates & & & & & \\
In-patients & $37(68.5)$ & $17(31.5)$ & $54(100)$ & 0.000 & 0.992 \\
Out-patients & $141(68.4)$ & $65(31.6)$ & $206(100)$ & & \\
\hline Total & $178(68.5)$ & $82(31.5)$ & $260(100)$ & & \\
\hline
\end{tabular}

\section{Discussion}

This study identified high rates of both MRSA and inducible clindamycin resistance among isolates from clinical specimens in FTHG, Nigeria. The prevalence of MRSA in this study (68.5\%) is higher than earlier studies of $12.5 \%$ and $8.0 \%$ from Gombe/North-eastern Nigeria $(12,13)$. This could be attributable to variation in time/methodology and changes in prescribing habits of clinicians and pattern of resistance among the microorganisms as reported in other studies across the globe $(15,16)$. However, differences (both higher and lower prevalence) with other Nigerian $(9,11)$ and foreign $(5,10,17)$ studies have been observed in both MRSA and inducible clindamycin resistance. These disparities are mainly due to variation in site and time of the studies while few are due to differences in the detection method. Most of the studies used phenotypic detection methods and also emphasized the need for routine reporting of these common MDROs to guide in rational use of antimicrobials and for good antimicrobial stewardship that will ensure patient safety $(18,19)$. Association of both MRSA and inducible clindamycin resistance with individual resistance to erythromycin and clindamycin as observed in this study is similarly reported by several other studies in Nigeria and other countries $(5,9-11,17,20)$. This further affirms the need for routine testing and reporting of common MDROs to appropriately guide prescribers in both targeted and empirical anti biotherapy, and to combat antimicrobial resistance for improved healthcare quality and patient safety.

The pattern of distribution of isolates among different specimens which revealed urine being the most frequent followed by swabs before seminal fluid, aspirates, and blood is a reflection of the frequency/predominance of these specimens in the laboratory requests. Similarly, several other studies have noted differences in pattern of distribution of isolates among different specimens 
but mostly without statistical significance and mainly due to disparities in location, patient distribution, type of study and practices among requesting physicians based on training and guidelines $(5,10,11,20)$. Variations in gender and age distribution did not also reveal any statistical significance probably due to similarities in habits/exposure with prevalence of the isolate among the different groups.

Although there is no significant difference in pattern of resistance between isolates from in and out-patients, most were from out-patients. This is probably because most microbiological investigations are requested at the points of hospital entry and in addition to poor utilization of clinical microbiology laboratory with overreliance on empirical anti biotherapy among Nigerian prescribers $(21,22)$. This also points to the danger of having MDROs circulating within the communities mainly due to poor regulation in the country, absence/ poor adherence to antibiotic guidelines among HCWs and misuse/abuse of antibiotics among especially general public in Nigeria $(22,23)$.

Phenotypic detection was used in this study largely because it is relatively cheap and easy to conduct on routine basis even in low resource settings like ours $(7,8)$. This can be timely advanced to requesting clinicians and other prescribers for better antimicrobial stewardship and improvement in patient safety. However genotypic detection has the additional advantage of identifying the resistant genes in the pathogens and has been reported by some studies mainly for research purposes in different parts of the world (24-26).

\section{Conclusion}

The prevalence of MDR Staphylococcus aureus (MRSA and iCR) is high in Gombe Nigeria. Significant association between MRSA and iCR and individual erythromycin/ clindamycin resistance was observed. Routine and timely reporting of findings to prescribers is necessary for rational anti biotherapy to combat AMR and improve IPC/patient safety. National and local guidelines/policies are important and should target both hospital and community-based utilization of antimicrobials with the concept of "one health" for better patient safety. Development of national/local guidelines, their wide dissemination is important and should be emphasized.

\section{Conflict of Interest}

The authors declare no conflict of interest.

\section{Aknowledgement}

We wish to acknowledge Kudi AA, Charanchi S, Hassan $D M, K$ wairanga $S$ and other members of the department of Medical Microbiology FTHG for their valuable contributions.

\section{References}

1. Casadevall A. Crisis in Infectious Diseases: 2 Decades Later. Clin Infect Dis 2017; 64(7):823-8.

2. Founou RC, Founou LL, Essack SY. Clinical and economic impact of antibiotic resistance in developing countries: A systematic review and meta-analysis. PLOS ONE 2017; 12(12): e0189621.

3. Guo Y, Song G, Sun M, Wang J, Wang Y. Prevalence and Therapies of Antibiotic-Resistance in Staphylococcus aureus. Front Cell Infect Microbiol 2020; [cited 2021 Jan 4]; Available from: https://www.frontiersin.org/articles/10.3389/fcimb. 2020.00107/full

4. Esguerra EM. Super Bugs and Antimicrobial Stewardship. Mo Med 2017;114(6):438-9.

5. Mama M, Aklilu A, Misgna K, Tadesse M, Alemayehu E. Methicillin- and Inducible Clindamycin-Resistant Staphylococcus aureus among Patients with Wound Infection Attending Arba Minch Hospital, South Ethiopia. International Journal of Microbiology 2019; [cited 2021 Jan 4]. p. e2965490.

6. Patel R, Fang FC. Diagnostic Stewardship: Opportunity for a Laboratory-Infectious Diseases Partnership. Clin Infect Dis 2018; 67(5):799-801.

7. Khan ZA, Siddiqui MF, Park S. Current and Emerging Methods of Antibiotic Susceptibility Testing. Diagnostics 2019; 9(2).

8. Bayot ML, Bragg BN. Antimicrobial Susceptibility Testing. In: StatPearls. Treasure Island (FL): StatPearls Publishing; 2020 [cited 2021 Jan 30].

9. Abdullahi N, Iregbu KC. Methicillin-Resistant Staphylococcus aureus in a Central Nigeria Tertiary Hospital. Ann Trop Pathol. 2018; 9(1):6.

10. Adhikari RP, Shrestha S, Barakoti A, Amatya R. Inducible clindamycin and methicillin resistant Staphylococcus aureus in a tertiary care hospital, Kathmandu, Nepal. BMC Infect Dis. 2017; 17(1):483.

11. Ifediora AC, Nwabueze RN, Amadi ES, Chikwendu Cl. Methicillin and Inducible Clindamycin-resistant Staphylococcus aureus Isolates from Clinical Samples in Abia State. Microbiol Res J Int. 2019; $1-9$.

12. Okon K, Uba ABP, Oyawoye OM, Yusuf IZ, Shittu AO, Blanc D. Epidemiology and characteristic pattern of methicillin-resistant Staphylococcus aureus recovered from tertiary hospitals in Northeastern, Nigeria. Int J Trop Med. 2011; 6:106-12.

13. Okon KO, Shittu AO, Kudi AA, Umar H, Becker K, Schaumburg F. Short Report: Population dynamics of Staphylococcus aureus from Northeastern Nigeria in 2007 and 2012. Epidemiol Infect. 2014; 142(8):1737-40.

14. Clinical and Laboratory Standards Institute (CLSI). M100 Performance Standards for Antimicrobial Susceptibility Testing. 2018. Cited 31/03/2021

15. Mhondoro M, Ndlovu N, Bangure D, Juru T, Gombe NT, Shambira G, et al. Trends in antimicrobial resistance of bacterial pathogens in Harare, Zimbabwe, 2012-2017: a secondary dataset analysis. BMC Infect Dis. 2019; 19(1):746

16. Zellweger RM, Basnyat B, Shrestha P, Prajapati KG, Dongol S, Sharma PK, et al. Changing Antimicrobial Resistance Trends in Kathmandu, Nepal: A 23-Year Retrospective Analysis of Bacteraemia. Front Med 2018; [cited 2021 Jan 28].

17. Mansouri S, Sadeghi J. Inducible Clindamycin Resistance in Methicillin-Resistant and-Susceptible Staphylococcus aureus Isolated From South East of Iran. Jundishapur Journal of Microbiology. 2014 [cited 2021 Jan 11]. Available from: https://sites.kowsarpub.com/jjm/articles/18765.html\#abstract

18. Guo S, Du W, Chen S, Guo X, Ju X. Exploring the Impact of the Rational Antibiotic Use System on Hospital Performance: The Direct Effect and the Spillover Effect. Int J Environ Res Public Health 2019; 16(18).

19. File TM, Srinivasan A, Bartlett JG. Antimicrobial Stewardship: Importance for Patient and Public Health. Clin Infect Dis 2014; 59(Suppl 3): S93-6.

20. Ghosh S, Banerjee M. Methicillin resistance \& inducible clindamycin resistance in Staphylococcus aureus. Indian J Med Res 2016; 143(3):362-4. 
21. Adisa R, Orherhe OM, Fakeye TO. Evaluation of antibiotic prescriptions and use in under-five children in Ibadan, Southwestern Nigeria. Afr Health Sci 2018; 18(4):1189-201.

22. Iregbu KC, Osuagwu CS, Umeokonkwo CD, Fowotade A, Ola-Bello OI, Nwajiobi-Princewill PI, et al. Underutilization of the Clinical Microbiology Laboratory by Physicians in Nigeria. Afr J Clin Exp Microbiol 2020; 21(1):53-9.

23. Chukwu EE, Oladele DA, Enwuru CA, Gogwan PL, Abuh D, Audu $\mathrm{RA}$, et al. Antimicrobial resistance awareness and antibiotic prescribing behavior among healthcare workers in Nigeria: a national survey. BMC Infect Dis 2021; 21(1):1-12.
24. Chen Z, Han C, Huang X, Liu Y, Guo D, Ye X. A molecular epidemiological study of methicillin-resistant and methicillin-susceptible Staphylococcus aureus contamination in the airport environment. Infect Drug Resist 2018; 11:2363-75.

25. Khashei R, Malekzadegan Y, Sedigh Ebrahim-Saraie H, Razavi Z. Phenotypic and genotypic characterization of macrolide, lincosamide and streptogramin $B$ resistance among clinical isolates of Staphylococci in southwest of Iran. BMC Res Notes 2018; [cited 2021 Jan 18]; Available from: https://www.ncbi.nlm.nih. gov/pmc/articles/PMC6180372/ 